\title{
Histone deacetylase (HDAC) inhibition improves myocardial function and prevents cardiac remodeling in diabetic mice
}

\author{
Youfang Chen', Jianfeng Du', Yu Tina Zhao', Ling Zhang ${ }^{2}$, Guorong LV', Shougang Zhuang ${ }^{2}$, Gangjian Qin ${ }^{4}$ \\ and Ting C Zhao ${ }^{1 *}$
}

\begin{abstract}
Background: Recent evidence indicates that inhibition of histone deacetylase (HDAC) protects the heart against myocardial injury and stimulates endogenous angiomyogenesis. However, it remains unknown whether HDAC inhibition produces the protective effect in the diabetic heart. We sought to determine whether HDAC inhibition preserves cardiac performance and suppresses cardiac remodeling in diabetic cardiomyopathy.

Methods: Adult ICR mice received an intraperitoneal injection of either streptozotocin (STZ, $200 \mathrm{mg} / \mathrm{kg}$ ) to establish the diabetic model or vehicle to serve as control. Once hyperglycemia was confirmed, diabetic mice received sodium butyrate (1\%), a specific HDAC inhibitor, in drinking water on a daily basis to inhibit HDAC activity. Mice were randomly divided into following groups, which includes Control, Control + Sodium butyrate (NaBu), STZ and STZ + Sodium butyrate (NaBu), respectively. Myocardial function was serially assessed at 7, 14, 21 weeks following treatments.

Results: Echocardiography demonstrated that cardiac function was depressed in diabetic mice, but HDAC inhibition resulted in a significant functional improvement in STZ-injected mice. Likewise, HDAC inhibition attenuates cardiac hypertrophy, as evidenced by a reduced heart/tibia ratio and areas of cardiomyocytes, which is associated with reduced interstitial fibrosis and decreases in active caspase-3 and apoptotic stainings, but also increased angiogenesis in diabetic myocardium. Notably, glucose transporters (GLUT) 1 and 4 were up-regulated following HDAC inhibition, which was accompanied with increases of GLUT1 acetylation and p38 phosphorylation. Furthermore, myocardial superoxide dismutase, an important antioxidant, was elevated following HDAC inhibition in the diabetic mice.
\end{abstract}

Conclusion: HDAC inhibition plays a critical role in improving cardiac function and suppressing myocardial remodeling in diabetic heart.

Keywords: HDAC, Diabetes, Myocardium, Heart failure, Apoptosis, GLUT

\section{Background}

Cardiovascular complications are the leading cause of diabetes-related morbidity and mortality worldwide [1]. Diabetes mellitus threatens to become a global health crisis; treatment of diabetes and its complications constitutes a major health care expenditure. A significant proportion of diabetic patients are known to develop

\footnotetext{
*Correspondence: tzhao@bu.edu

${ }^{1}$ Department of Surgery, Boston University Medical School, Roger Williams Medical Center, Boston University, 50 Maude Street, Providence, RI 02908, USA

Full list of author information is available at the end of the article
}

diabetic cardiomyopathy, with a high incidence of congestive heart failure [2-4]. For example, in patients with diabetes mellitus, both the prevalence and severity of manifestations of coronary artery disease are increased compared with non-diabetic subjects. Experimental evidence of diabetic cardiomyopathy has been accumulated in disease models with both type 1 and type 2 diabetes [5-8]. Evidence from STZ-induced hyperglycemia displayed decreased cardiac function, a reduction in cardiac mass over time, and a variety of morphological changes, including cardiac hypertrophy, myofibril depletion, interstitial fibrosis, and microangiopathy [9-12]. 
Histone acetyltransferases (HAT) and HDAC have recently garnered attention because they have emerged as important mechanisms in the regulation of a variety of cellular responses. Histone acetylation is mediated by HAT, which results in the modification of the structure of chromatin leading to nucleosomal relaxation, and altered transcriptional activation. In contrast, the reverse reaction is mediated by HDACs which induce deacetylation, chromatin condensation, and transcriptional repression. The acetylation status of histone tails is determined by the interplay between HATs and HDACs [13-15]. Recent observation indicates that regulation of acetylation status mediates proteolytic function in diseased myocardium from human and animal models [16]. These recent studies have indicated the critical role of HDACs in modulating myocardial ischemia/reperfusion injury, cardiac hypertrophy, and skeletal myogenesis [17-22]. Inhibition of HDACs using small molecules is regarded to be one of the most promising approaches for many pathological disorders.

Our studies have demonstrated that inhibition of HDACs plays a critical role in the prevention of myocardial damages, mediation of cardiogenesis, and stimulation of myocardial repair [23-27]. In addition, the function of HDAC was reported to be associated with the pathological process in diabetic status and/or heart failure [28, 29]. However, it remains unknown whether HDAC inhibition could produce a protective effect against myocardial dysfunction in diabetic mice. In this present study, we utilized the STZ-induced diabetic model to define whether HDAC inhibition could preserve cardiac performance and attenuate cardiac remodeling.

\section{Methods}

\section{Animals}

Two-month old ICR male mice were purchased from Charles River Laboratories (Wilmington, MA, USA). All animal experiments were conducted under a protocol approved by the Institutional Animal Care and Use Committee of Institute, which conforms to the Guide for the Care and Use of Laboratory Animals published by the US National Institutes of Health (NIH Publication No. 85-23, revised 1996).

\section{Experiment protocols}

Two month-old mice were made diabetic by intraperitoneal injection of a single dose of freshly prepared STZ solution $(200 \mathrm{mg} / \mathrm{kg}$ body wt dissolved in citrate buffer, $\mathrm{pH}$ 4.5) following overnight fasting [30]. Diabetic status was determined by measuring blood glucose concentration. Another group of mice was injected with vehicle $(0.1 \mathrm{~mol} / \mathrm{l}$ citrate buffer, $\mathrm{pH} 4.5)$ to serve as a control. Mice were randomly divided into four groups: Control group: mice only received an injection of vehicle (citrate buffer); Control + NaBu group: mice received sodium butyrate (1\%), a specific HDAC inhibitor, in drinking water on a daily basis; $S T Z$-treated group: mice received intraperitoneal injection of STZ; $S T Z+N a B u$ group: mice received intraperitoneal injection of STZ injection followed by sodium butyrate (1\%), in drinking water on a daily basis. Tail vein blood glucose samples were measured using One Touch II Glucometer (Lifescan, Inc., Milpitas, CA, USA) to confirm the induction of diabetes. All animals were euthanized at 21 weeks after injection of STZ.

\section{Echocardiography}

Echocardiographic parameters were accessed before and 7,14 , and 21 weeks after the sodium butyrate treatments. Echocardiography was performed to evaluate left ventricular (LV) functions using an Acuson Sequoia C512 system (Siemens Helathcare, PA, USA) equipped with a 15L8 linear array transducer. Mice were placed in supine position on a heating pad after being anesthetized with $1.5 \%$ isoflurane mixed with oxygen. Pre-warmed ultrasound gel was applied on the chest throughout the measurements. At the signal depth of $25 \mathrm{~mm}, 2-\mathrm{D}$ B-mode and $\mathrm{M}$-mode images were recorded on short axis views at the level of the papillary muscles. The following parameters were measured on the M-mode tracings and averaged from 3 to 6 cardiac cycles: left ventricular internal dimension-diastole (LVID;d), left ventricular internal dimension-systole (LVID;s), ejection fraction (EF), fraction shortening (FS), left ventricular posterior wall thickness in end-diastole (LVPW;d), and end-systole (LVPW;s). Data were calculated with accompanying software.

\section{Immunohistochemistry}

Tissue sections were de-paraffinized for $30 \mathrm{~min}$ at $70^{\circ} \mathrm{C}$ and subsequently immersed in xylene and ethanol at decreasing concentration. Immunostaining was performed as described previously [27]. All de-paraffinized tissue sections went through antigen retrieval by boiling of slides at $100^{\circ} \mathrm{C}$ for $1 \mathrm{~h}$. Wheat germ agglutinin (WGA) staining was carried out to measure cell size. The outline of myocytes was traced in the LV of each animal, using NIH Image $J$ software to determine myocyte cross-sectional area. A value from each heart was calculated by the measurements of approximately 400-600 cells in a remote area from 5 randomly selected image areas in an individual heart. For evaluation of interstitial fibrosis, Picrosirius red staining of cardiac sections was conducted. Interstitial collagen was examined from five randomly selected regions from each tissue section using an Olympus BX51 microscope (Olympus, Center Valley, PA, USA). For $\alpha$-smooth muscle actin ( $\alpha$-SMA) and cluster of differentiated CD31 
immunostaining, cardiac sections were incubated with primary antibodies including CD31 (Millipore, Billerica, MA, USA) and anti- $\alpha$-SMA (Sigma, St. Louis, MO, USA) overnight at $4^{\circ} \mathrm{C}$. Signals were visualized by incubation with the corresponding secondary antibodies including goat-antirat-Cy3 and goat-anti-mouse-Cy3 (Life Technologies, Carlsbad, CA, USA) at room temperature for $1 \mathrm{~h}$. Nuclei were stained with $4^{\prime}$, 6-diamidino-2-phenylindole (DAPI).

\section{Terminal deoxynucleotidyl transferase dUTP nick end labeling (TUNEL) assay}

De-paraffinized sections were processed for a TUNEL assay using a $\mathrm{TACS}^{\circledR} \mathrm{TdT}$ In Situ Apoptosis Detection Kits (Trevigen, Gaithersburg, MD, USA) following the manufacturer's instructions. TUNEL positive cells were observed using confocal laser scanning microscopy LSM 700 (Carl Zeiss). The numbers of TUNEL positive cells were determined and were normalized to the tissue area. DAPI was used to counter stain for nuclei.

\section{Immunoblotting and immunoprecipitation}

Heart tissues were homogenized on ice in RIPA buffer with Halt protease and phosphatase inhibitor cocktail. Proteins $(50 \mu \mathrm{g} /$ lane $)$ were resolved by SDS gel and transferred onto PVDF membranes. The membranes were then blocked with 5\% powdered milk in TBST for $1 \mathrm{~h}$ and were subsequently probed with primary antibodies overnight at $4^{\circ} \mathrm{C}$. The following primary antibodies were used in this study: HDAC4, acetylated-Lysine, phosphorylated p38, and active casepase-3 from Cell Signaling (Danvers, MA, USA); GLUT 1, GLUT4, p38, SOD1, and $\beta$-actin were from Santa Cruz Biotechnology (Dallas, TX, USA). Horseradish peroxidase-conjugated monoclonal antibodies (1: 2,000) were used for chemiluminescence detection.

For immunoprecipitation, samples were pre-cleared prior to immunoprecipitation to reduce the amount of non-specific contaminants. The EZView red protein A affinity gel (Sigma-Aldrich, St.Louis, MO, USA) was incubated with myocardial lysates for $60 \mathrm{~min}$ at $4^{\circ} \mathrm{C}$. Samples were centrifuged, and supernatants were obtained. Proteins were incubated with the indicated primary antibodies at $4^{\circ} \mathrm{C}$ overnight. Beads were added to lysate plus antibody mix, and proteins were further incubated for $2 \mathrm{~h}$ at $4^{\circ} \mathrm{C}$. In addition, IgG was also used as immunoprecipitation control and non-immunoprecipitated lysate was used as a control for detected molecular weight. After incubation, samples were washed with RIPA buffer five times, and proteins were eluted with $4 \times$ loading buffer by boiling and subjected to SDS-PAGE.

\section{HDAC activity measurement}

Myocardial HDAC activity was measured as described preciously in detail [25].

\section{Statistical analysis}

All data are expressed as mean \pm SEM. Differences among multigroups were analyzed by one-way analysis of variance (ANOVA), followed by Bonferroni correction. A probability of $p<0.05$ was considered to be a significant difference.

\section{Results}

\section{Induction of hyperglycemia}

A single injection of STZ (200 mg/kg) was used to induce diabetes in ICR mice. At 1 week after the injection, the fasting blood glucose levels of $>450 \mathrm{mg} / \mathrm{dl}$ were detected in all mice that received STZ injection. Approximately $20 \%$ mice did not survive to sustain the toxicity of STZ by day 14 . The surviving mice had blood glucose levels of $>450 \mathrm{mg} / \mathrm{dl}$ throughout the study period. As shown in Fig. 1, sodium butyrate treatment did not lower the blood glucose level. The two diabetic groups showed no difference in blood glucose level by the end of the study period.

\section{Ventricular function}

Serial echocardiography was performed immediately before and up to 21 weeks after treatments. As shown in Fig. 2, STZ induced diabetic mice displayed cardiac dysfunction, as indicated by the reduction of both EF and FS as compared to Control group. However, administration of sodium butyrate resulted in improvements in EF and FS as compared with STZ group (Fig. 2a, b). The representative images of $\mathrm{M}$-mode among groups are shown in Fig. 2c. Furthermore, an increase in left ventricular internal dimension (LVID) was observed in STZ-treated group as compared with Control and

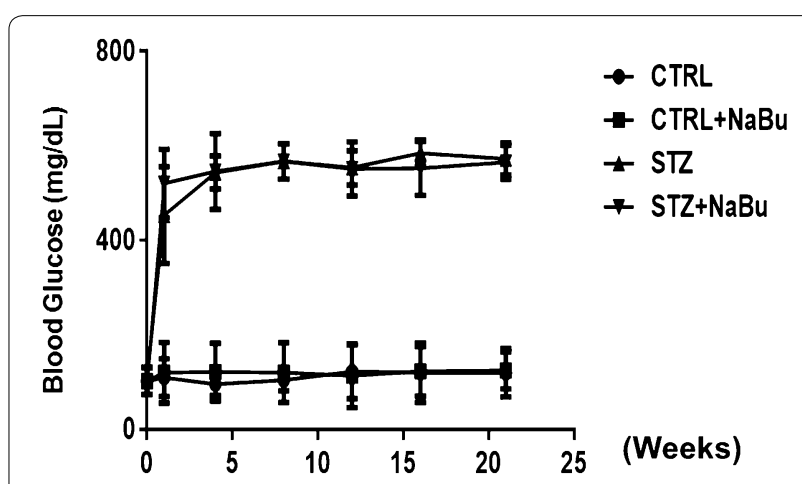

Fig. 1 The time course of measuring the concentration of blood glucose. The peripheral blood concentration was measured on weekly basis. After overnight fasting, blood samples were collected from the tail tip vein on mice. Measurements were performed on control, STZ and STZ+ Sodium butyrate treatment groups, respectively. Mice with blood glucose readings of $>250 \mathrm{mg} / \mathrm{dl}$ were diagnosed diabetic. Values represent mean \pm SEM ( $n=5-7$ per group); CNTL control; STZ streptozotocin; $\mathrm{NaB}$ sodium butyrate. 


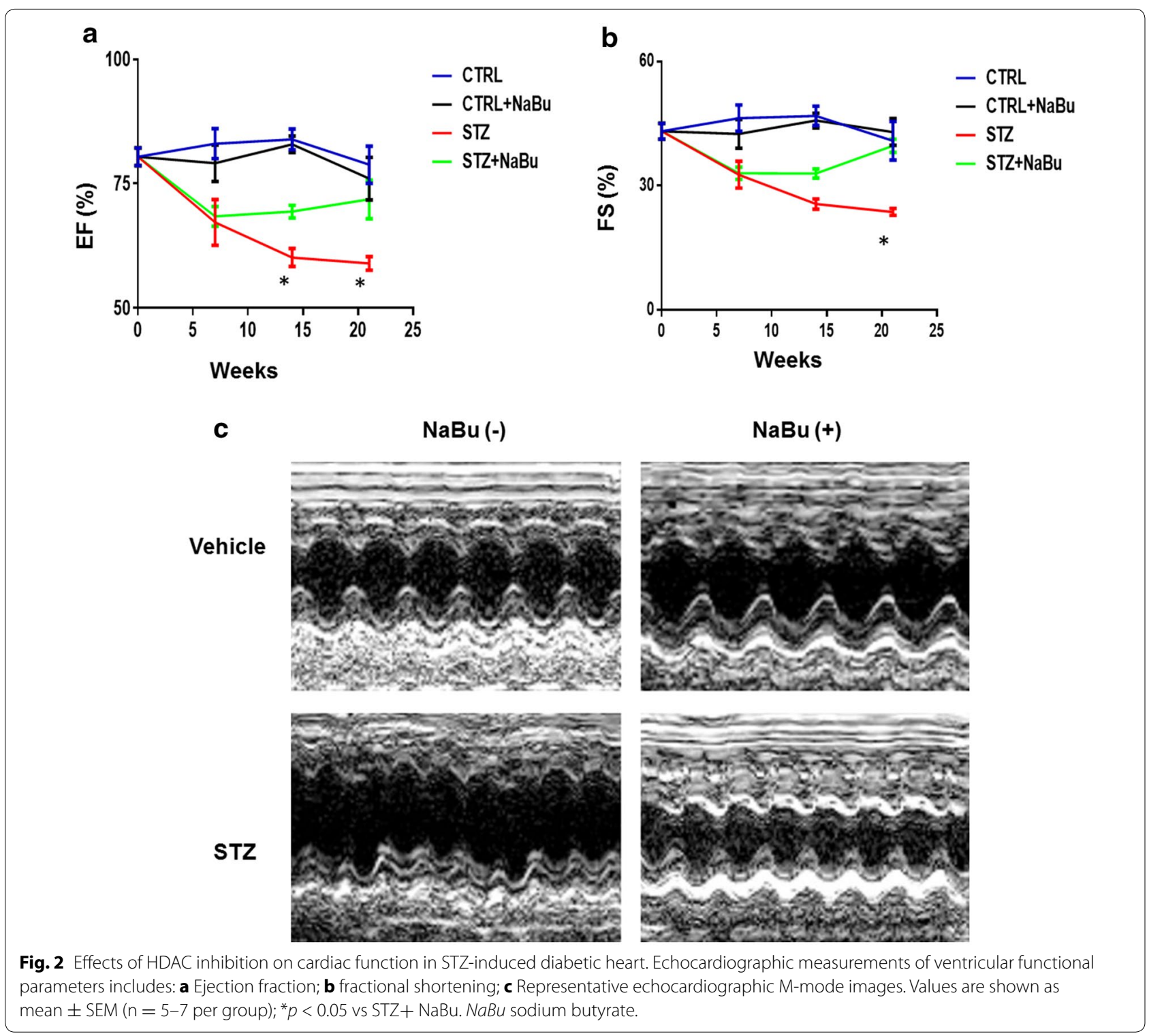

Control $+\mathrm{NaBu}$ groups starting at 7 weeks following STZ injection throughout the 21-week period. LVID;s was significantly reduced in sodium butyrate-treated STZ mice as compared with STZ diabetic mice without treatment (Additional file 1: Table S1). In addition, STZ-induced diabetic mice demonstrated an increase in LVPW, which was also attenuated by treatment of animals with sodium butyrate (Additional file 1: Table S1).

\section{Cardiac remolding and interstitial collagen deposition}

The ratio of heart weight to tibia length was used to evaluate the hypertrophic response. STZ injection resulted in an increase in the heart weight/tibia length ratio as compared to control hearts. Treatment of STZ mice with sodium butyrate significantly reduced the heart weight/tibia length ratio (Fig. 3a). In addition, STZ treatment caused a significant increase in heart weight/body weight ratios (Additional file 1: Table S2), which was prevented by treatment with sodium butyrate. STZ-induced diabetic mice WGA staining was performed to access the cross sectional cardiomyocyte size. As shown in Figs. 3b, c, the STZ-treated mice showed an increase in cross-sectional cardiomyocyte diameters as compared with control groups. However, the cross-sectional cardiomyocyte diameter was significantly reduced in STZ mice receiving sodium butyrate as compared with STZ mice only (Fig. 3c). As 


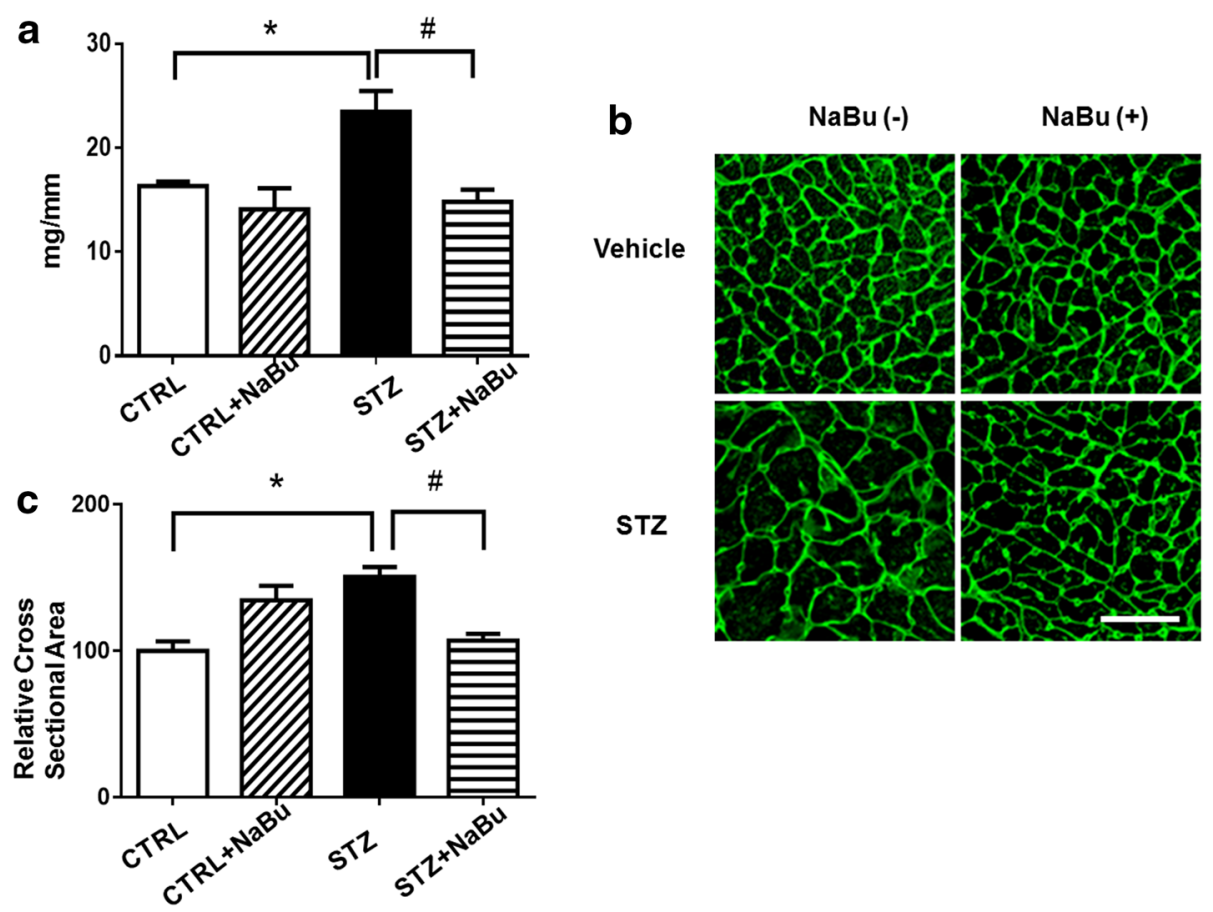

Fig. 3 Effects of HDAC inhibition on attenuating cardiac remodeling in STZ-induced diabetic heart. a The ratio of heart weight to tibia length. $\mathbf{b}$ Representative images of WGA staining in the MI hearts. c Quantitative analysis of myocyte cross-sectional area. Values are shown as mean \pm SEM ( $n=5$ per group); ${ }^{*} p<0.05$ vs CTRL, ${ }^{\#} p<0.05$ vs STZ+ NaBu. NaBu sodium butyrate. Scale bar $100 \mu \mathrm{m}$.

shown in Fig. 4a, b, the collagen content was significantly increased in STZ group as compared with control groups, but administration of sodium butyrate led to a reduction in interstitial collagen deposition in the myocardium as compared with STZ-treated mice alone although there is no statistically difference between the two diabetic groups.

\section{Inhibition of HDACs resulted in up-regulation of GLUT1} and 4 and acetylation of GLUT1

When we measured HDAC activity in the diabetic myocardium, HDAC activity demonstrated an increase in STZ-induced diabetic heart, but HDAC activity was significantly reduced following the treatment of animals with sodium butyrate (Fig. 5a). In addition, as shown

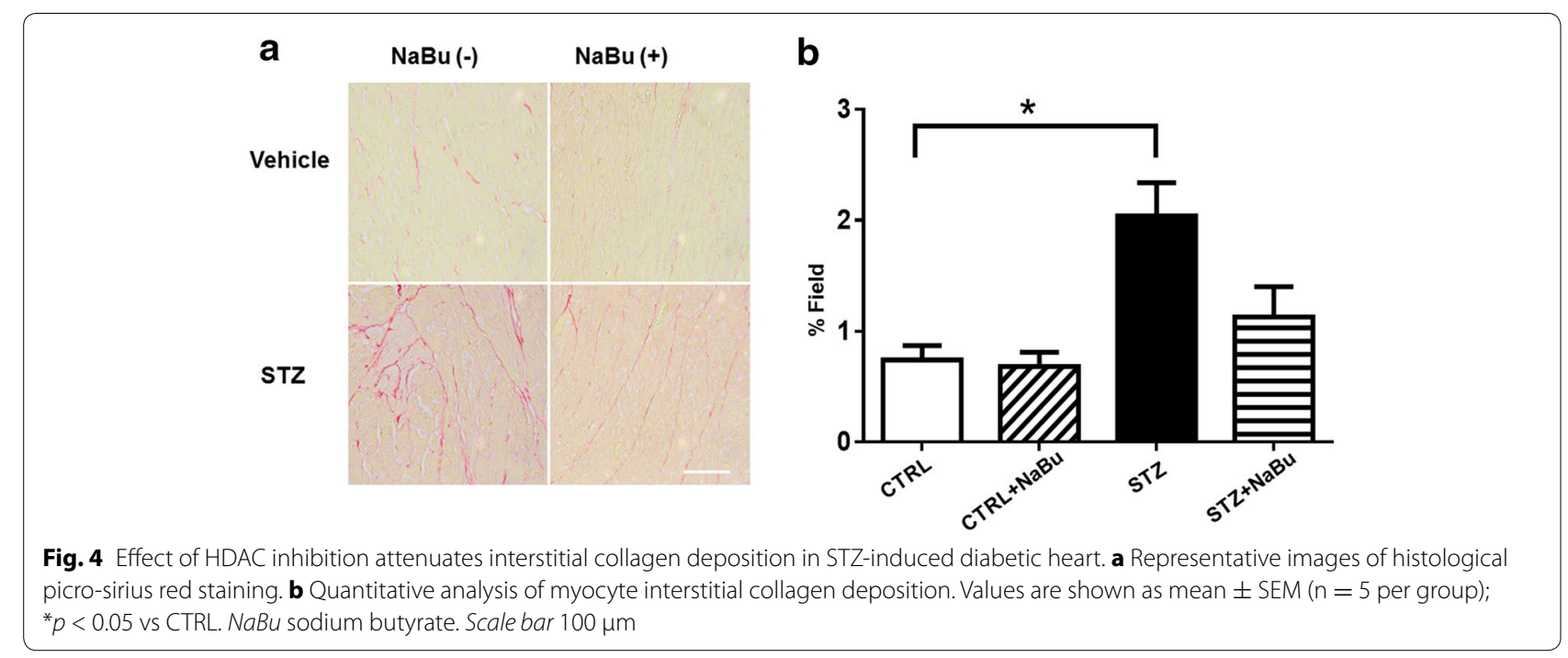




\section{"1-1}

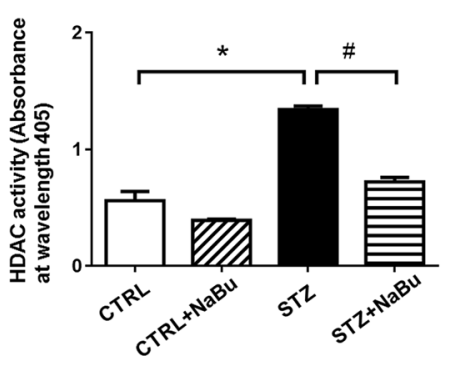

b

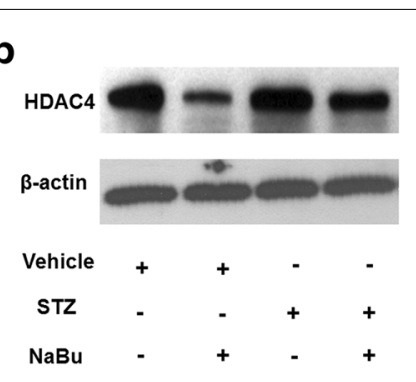

C

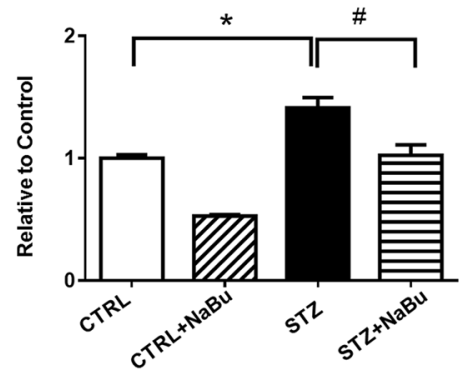

d

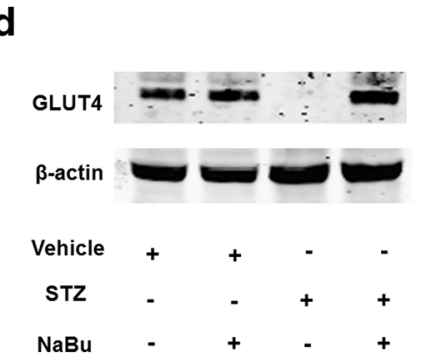

f
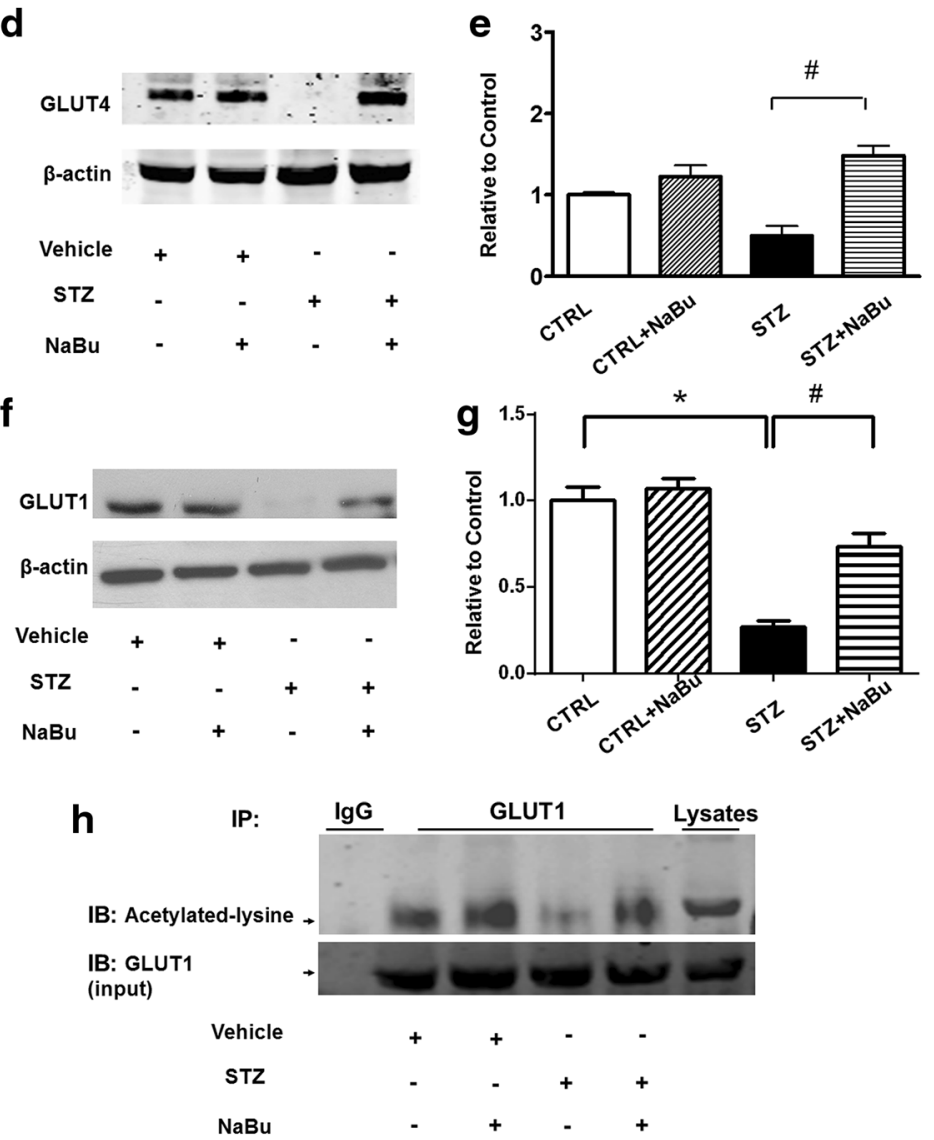

Fig. 5 HDAC inhibition decreases HDAC activity and increases GLUT1 and GLUT4. a Measurement of HDAC activity in diabetic myocardium from different groups; b representative Western blot of HDAC4 proteins in myocardium; c densitometric analysis of Western blot of HDAC4 protein levels; d representative Western blot of GLUT4 proteins in myocardiums; e densitometric analysis of GLUT4 proteins in myocardium; $\mathbf{f}$ representative Western blot of GLUT1 proteins in myocardium; $\mathbf{g}$ densitometric analysis of GLUT1 proteins in myocardium; $\mathbf{h}$ GLUT1 acetylation in diabetic myocardium. IB immunoblot; IP immunoprecipitation; IgG serves as IP control; Iysates from myocardium serve as signal control of detected proteins. The densitometric signal was normalized to the control group and expressed as a percentage. Values are shown as mean \pm SEM $\left(n=3\right.$ per group); ${ }^{*} p<0.05$ vs CTRL, ${ }^{\#} p<0.05$ vs STZ+ NaBu. NaBu sodium butyrate. 
in Fig. 5b, c, treatment of mice with sodium butyrate decreased the expression level of HDAC4 in both Control and STZ-treated groups. Other HDAC isoforms were not changed in the diabetic myocardium between groups (Additional file 1: Figure. S1). The glucose transporter signaling pathway was further confirmed. As shown in Fig. 5d-g, STZ induced a down-regulation of GLUT1 and GLUT4 protein levels in STZ heart as compared with Control groups. However, HDAC inhibition resulted in up-regulations in both GLUT4 and GLUT1 in STZ heart, which restored the signal to the same level as the control group. Notably, GLUT1 was subjected to regulation by acetylation. As shown in Fig. 5h, HDAC inhibition increased the level of acetylated GLUT1 in sodium butyrate-treated STZ mice as compared with STZ alone. However, HDAC inhibition did not result in a significant increase in acetylated GLUT4 although we noted there was a detectable level of acetylated GLUT4. Interestingly, as shown in Fig. 6, we found that the diabetic myocardium demonstrated a decrease in phosphorylated p38 level, but HDAC inhibition resulted in a significant increase in phosphorylation of p38.

\section{Anti-apoptotic effects of HDAC inhibition in the diabetic myocardium}

The apoptotic marker active caspase-3 was examined in the myocardium. As shown in Fig. 7a, active caspase-3 was increased in STZ group as compared with samples from Control groups. Densitometric analysis confirms that the administration of sodium butyrate significantly reduced activated caspase-3 in STZ mice (Fig. 7b). As shown in Fig. 7c, $\mathrm{d}$, TUNEL analysis indicates that STZ-injection increased the number of TUNEL-positive nuclei, but HDAC inhibition reduced apoptotic signals in the diabetic heart.

\section{HDAC inhibition increases endogenous antioxidant enzyme SOD1}

Superoxide dismutase has been implicated in protective effects against myocardial ischemic injury. The levels of SOD1 in the myocardium were examined. As shown in Fig. 8a, all mice treated with sodium butyrate showed an increase in SOD1 level as compared to mice without sodium butyrate treatment. Densitometric analysis also indicates significant increases in SOD1 1evel following HDAC inhibition (Fig. 8b). In addition, the diabetic myocardium demonstrated an increase in superoxide production, which was suppressed by HDAC inhibition (Additional file 1: Figure S2).

\section{HDAC inhibition induces angiogenesis in the diabetic myocardium}

As shown in Fig. 9a, CD31 positive capillary density was decreased in STZ mice. Administration of sodium butyrate substantially increased the capillary density in myocardium from STZ mice. Furthermore, we examined $\alpha$-SMA positive microvessels in STZ-induced diabetic mouse hearts. Figure 9c shows $\alpha$-SMA staining in the heart sections from all treatment groups. There is a significant decrease in $\alpha$-SMA-positive microvessels in STZ mice as compared to Control group, but HDAC inhibition resulted in a marked increase in microvessels. As shown in Fig. 9b, d, HDAC inhibition increased CD31 and $\alpha$-SMA positive vessels in the diabetic myocardium.

\section{Discussion}

\section{Salient findings}

Our study is the first to document that HDAC inhibition preserves cardiac performance and suppresses cardiac remodeling in diabetic cardiomyopathy. Specifically,

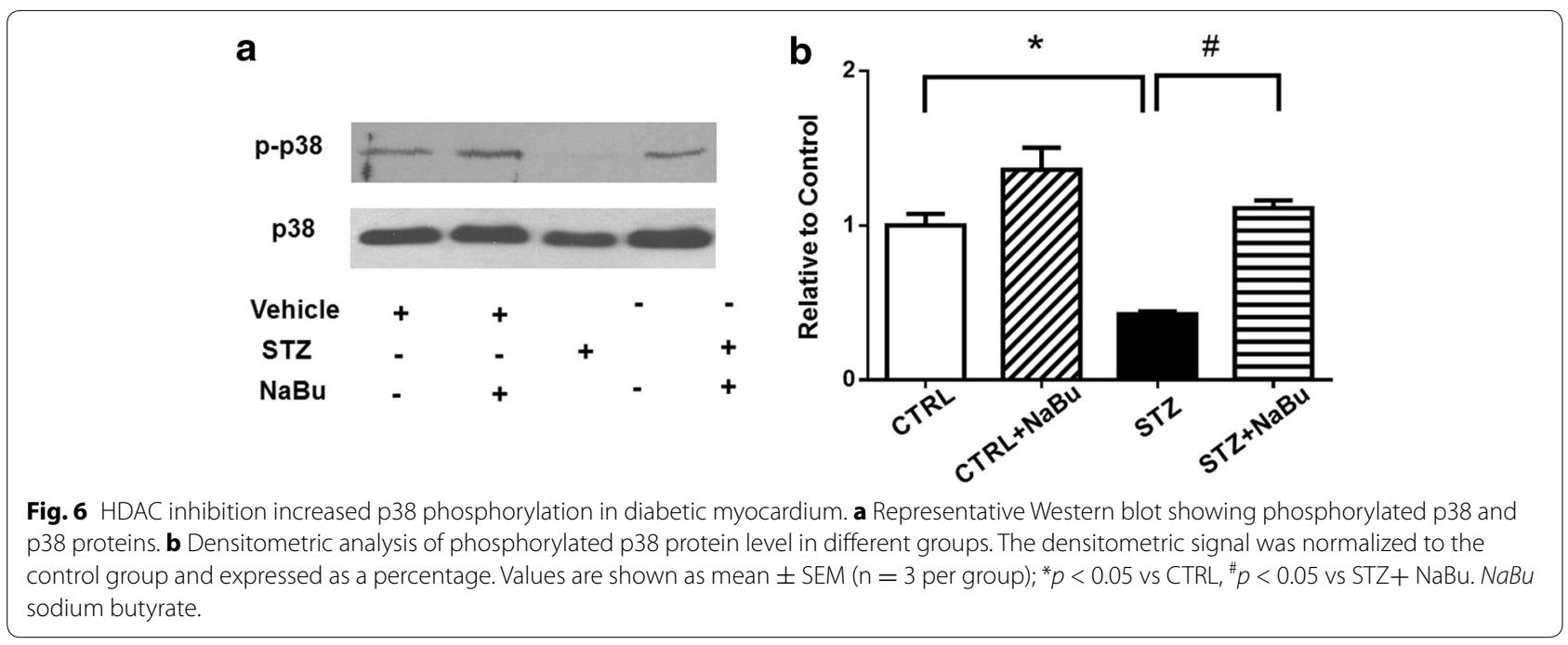




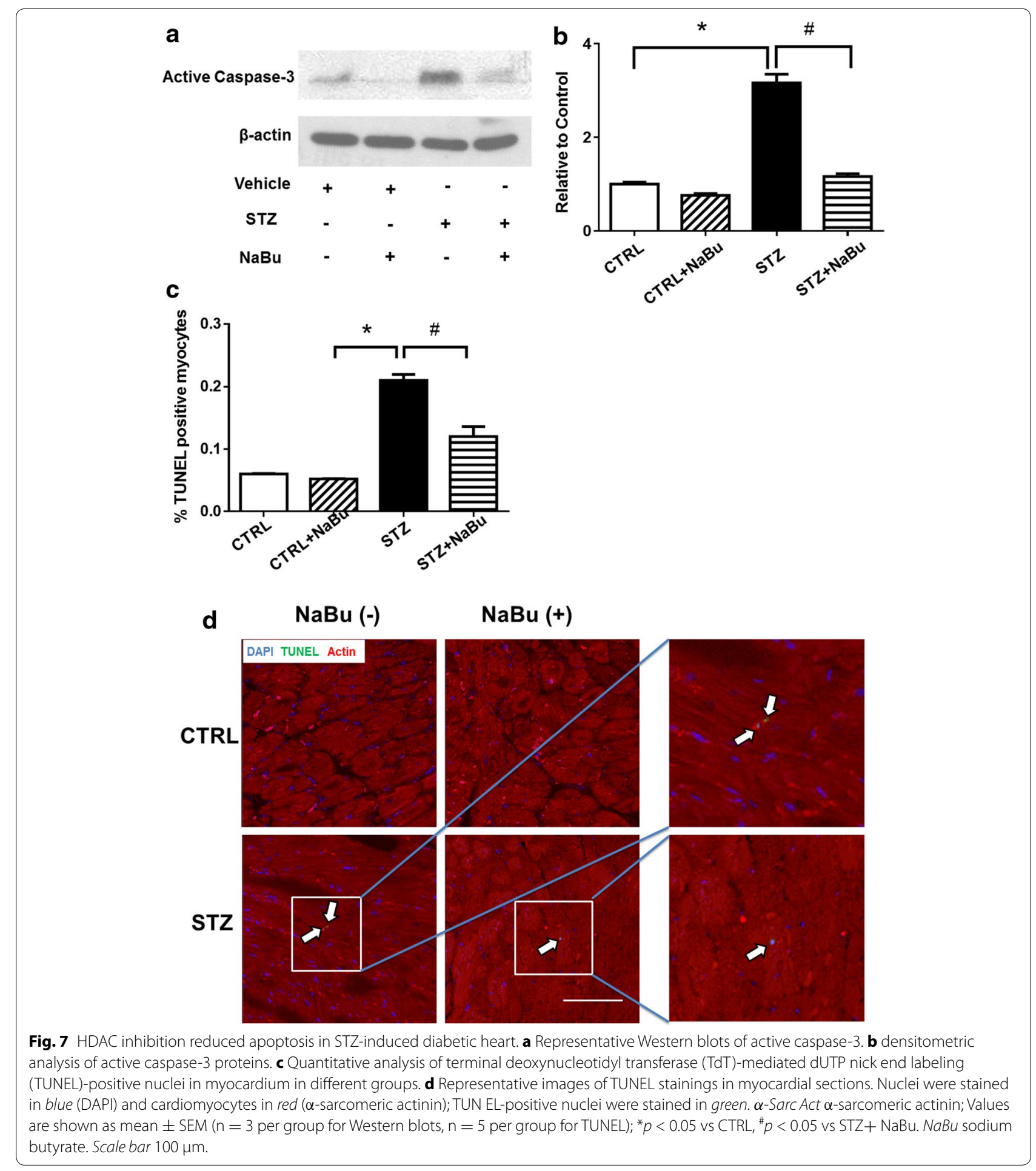

(1) Serial echocardiographic evaluation indicates that HDAC inhibition resulted in the preservation of ventricular function in STZ-induced diabetic heart; (2) HDAC inhibition plays a profound effect in suppressing interstitial fibrosis and attenuating myocyte hypertrophy in the diabetic myocardium; (3) HDAC inhibition mitigated the frequencies of apoptosis in the diabetic myocardium by decreasing active caspase 3 and TUNEL positive signals; (4) HDAC inhibition resulted in the increase of SOD1, GLUT1, and GLUT4 protein levels in diabetic hearts, 

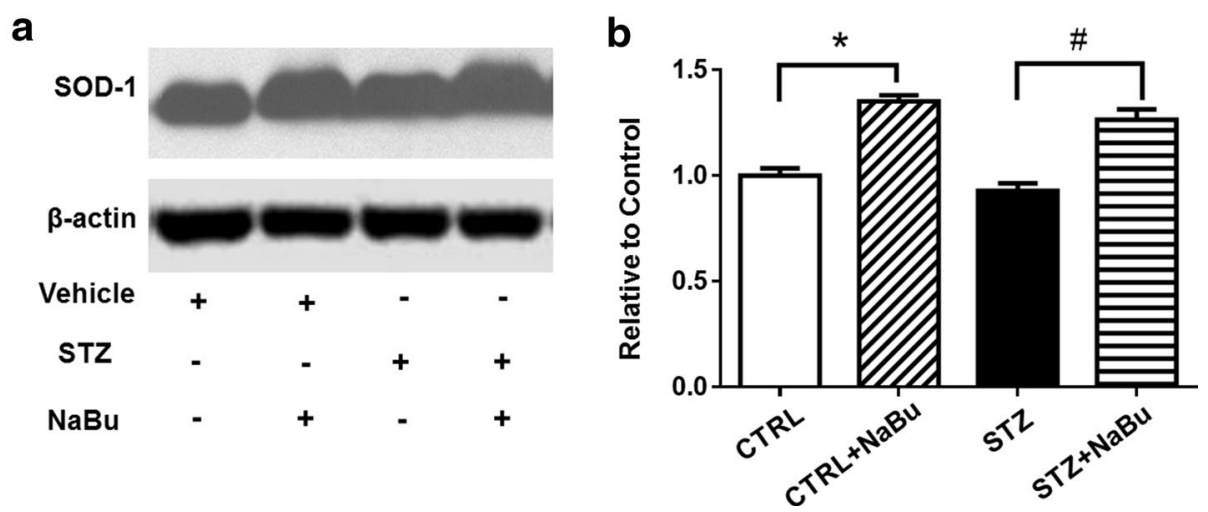

Fig. 8 HDAC inhibition reduced SOD1 expression in myocardium. a Representative Western blots of active SOD1. b Densitometric analysis of Western blot results. Values are shown as mean $\pm \operatorname{SEM}\left(n=3\right.$ per group); ${ }^{*} p<0.05$ vs CTRL, ${ }^{\sharp} p<0.05$ vs STZ+ NaBu. NaBu sodium butyrate.
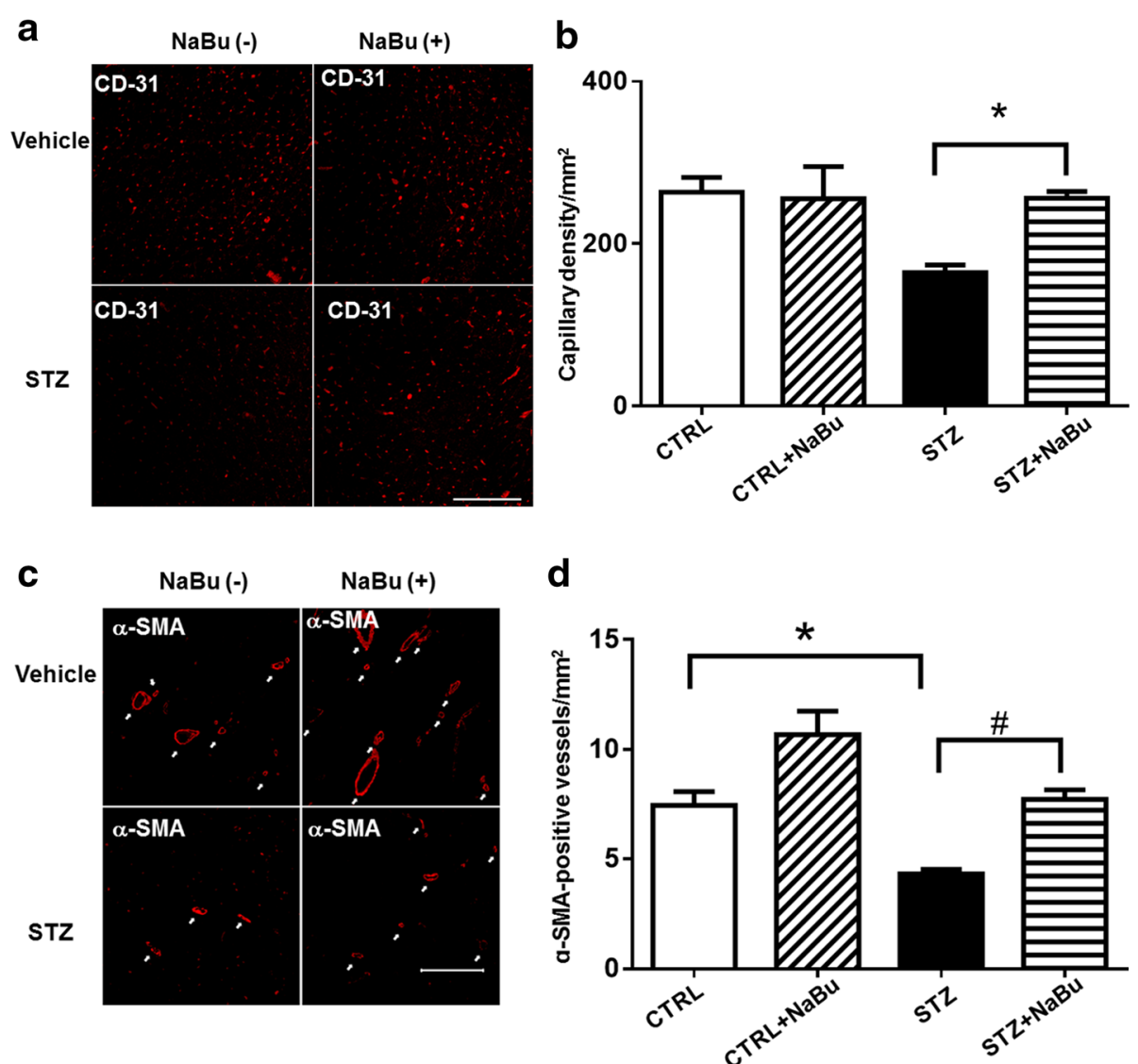

Fig. 9 HDAC inhibition promotes angiogenesis in STZ-induced diabetic heart. a, c Representative images of CD31 and $\alpha$-SMA staining, respectively. $\mathbf{b}, \mathbf{d}$ quantitative analysis of angiogenetic response. Results were indicated by the number of CD31- or $\alpha$-SMA-positive cells per mm². Values are shown as mean \pm SEM ( $n=3$ per group); ${ }^{*} p<0.05$ vs CTRL, ${ }^{\#} p<0.05$ vs STZ+ NaBu; NaBu sodium butyrate. Scale bar $100 \mu m$ for CD31, $200 \mu m$ for $\alpha-S M A$. 
and acetylation of GLUT1 was elevated following HDAC inhibition; (5) Diabetic hearts exhibited significant decreases in CD31 and $\alpha$-SMA positive microvessels, which was prevented following HDAC inhibition.

Recent evidence has indicated a genetic association between diabetes and HDACs. HDAC inhibitors promote $\beta$-cell development and function that positively affect diabetic microvascular complications [31]. Ventricular hypertrophy in diabetic hearts was mitigated by inhibition of HDACs [32]. Transient hyperglycemia is considered to promote gene-activating epigenetic changes critical in the progression of vascular complications [33]. Therefore, the function of epigenetics in the development of diabetes is largely recognized [34]. However, its implications in diabetes-associated cardiomyopathy remain to be determined. Our study showed that HDAC inhibition attenuates progressive dysfunction, suggesting that HDACs play an important role in controlling the progression of heart failure.

Clinical studies demonstrate that diabetes mellitus increased the susceptibility of the myocardium to ischemic injury $[35,36]$. In the present study, when we employed a STZ-induced diabetic model [37], we have demonstrated that the diabetic myocardium (DM) presents with progressive LV systolic failure following STZ treatment for 21 weeks, which is consistent with observations in the development of cardiac dysfunction in STZ-induced diabetic rats [38]. This was supported by an observation that HDAC inhibition prevented the heart from effects of diet-induced obesity and insulin resistance in mouse models [39]. Further estimation of diastolic function in this model will determine whether diabetic cardiomyopathy developed. In addition, the low dose of STZ could be used as a diabetogenic method to induce diabetes to avoid the high rate of mortality caused by high dose of drug. Treatment of sodium butyrate also decreased HDAC activity and HDAC4 levels in DM myocardium, which is in agreement with our recent observations that specific inhibition of HDAC4, by reducing HDAC4 protein, promotes stem cell-derived myocardial repair [40]. Furthermore, we could not find significant changes in other HDAC isoforms following HDAC inhibition in diabetic myocardium. Myocardial enhancing factor 2 (MEF2) is reported to be associated with class II HDACs to mediate cardiac growth and remodeling [41]. We found that HDAC inhibition only slightly increased MEF2 protein levels in STZ-induced myocardium (not shown), implying that MEF2 may not be a major target following HDAC inhibition in STZ-induced diabetic myocardium. An important pathological feature of diabetic cardiomyopathy is cardiac hypertrophy [42]. In the initial stage, this hypertrophic cardiomyopathy may be an adaptive response responsible for enhancing cardiac performance [43]. However, sustained hypertrophic growth of the myocardium may be associated with the occurrence of myocardial remodeling [20]. HDAC inhibitors blocked cardiac hypertrophy induced by angiotensin II infusion and aortic banding (18.19). We observed that HDAC inhibition remarkably prevented these hypertrophic features in DM. The anti-hypertrophic effect of HDAC inhibition in the DM heart is well mirrored by our recent studies in which cardiac hypertrophy was mitigated in the infarcted hearts following global HDAC inhibition or infarcted hearts engrafted with cardiac stem cells treated with HDAC inhibitors [27].

Myocardial fibrosis is another important hallmark of diabetic cardiomyopathy, and it is also featured by the accumulation of interstitial collagens in the hearts [44]. The present results demonstrate that interstitial collagen in DM hearts was reduced after treatment with HDAC inhibition, indicating the function of HDAC in preventing interstitial fibrosis. Another finding is an increase of myocyte apoptosis in DM hearts. Although the significance of myocyte apoptosis in diabetic myocardium remains speculative, progressive loss of myocytes could exacerbate cardiac dysfunction and structure deterioration.

We reported that HDAC inhibition protects the heart against ischemia/reperfusion through blocking of reactive oxidant species [25]. Oxidative stress has been suggested by several studies to underlie hyperglycemiainduced myocardial cell deaths [45-47]. High glucoseinduced cardiomyocyte apoptosis is associated with the generation of reactive oxygen species [48], and normalization of SOD1 activity was associated with consequent bolstering of anti-oxidant defenses in diabetes [49].The present study shows that SOD1 was markedly increased by HDAC inhibition, suggesting that the increased antioxidant stress elicited by HDAC inhibition accounts for the suppression of myocardial remodeling in the diabetic heart. In addition, the production of superoxides was increased in the diabetic myocardium, but HDAC inhibition attenuated the superoxide production of diabetic hearts, revealing a link between HDAC inhibition and suppression of superoxide.

It is known that pathological cardiac hypertrophy with reduced contractility is accompanied by impaired coronary angiogenesis [50]. The reductions in capillary and arteriolar densities were shown in the myocardial infarction model of diabetic conditions [51, 52]. Our study demonstrates a significant reduction in microvessel density in diabetic myocardium, but vascular growth was stimulated by treatment with HDAC inhibition. This increase in microvessel density following HDAC inhibition in diabetic myocardium may also be attributable to the improvement in cardiac function and attenuation of remodeling. 
Two glucose transporter proteins (GLUT1 and GLUT4) were reported to be reduced in the diabetic myocardium [53]. We have shown that GLP-1-induced myocardial protection is associated with the increase of GLUT4 [53]. In this study, both GLUT1 and GLUT4 were decreased in the diabetic myocardium, but the decrease of both GLUT1 and GLUT4 was prevented by HDAC inhibition. Interestingly, our results show that acetylation of GLUT1 was increased by HDAC inhibition. However, HDAC inhibition did not result in a significant change in the acetylation of GLUT4 (data not shown). This suggests that acetylation of GLUT1 and GLUT4 may respond differently following HDAC inhibitions or depend on the magnitudes of HDAC inhibition. Although the content of GLUT1 and GLUT4 were increased by HDAC inhibition in the diabetic animals, blood glucose level was not reduced by HDAC inhibition in this studies. It is likely that HDAC inhibition could not reduce the peripheral glucose concentration, but instead modulate insulin resistance in the diabetic status, which will be an interesting subject to investigate in the future. It is interesting to elucidate whether acetylation of GLUT1 could mediate its physiological functions in the future. In addition, we have shown that $\mathrm{p} 38$ phosphorylation was associated with cardioprotection induced by glucagon-like peptide (GLP1) in myocardial ischemia and reperfusion [54]. The present study again reveals that HDAC inhibition increased p38 phosphorylation in diabetic hearts. The future study may attempt to document whether there exists a direct relationship between p38 phosphorylation and GLUT1 acetylation in diabetic hearts. It is not clear whether the PI3 kinase/Akt-1 signaling pathway is also involved in the protective effects induced by HDAC inhibition. On the other hand, mitochondrial oxygen consumption was impaired in the diabetes related mouse model [55].Our previous observation also indicates that stimulation of GLP-1R could increase mitochondrial oxygen consumption in myoblasts exposed to hypoxia/reoxygenation [56]. It is not clear whether HDAC inhibition could also modulate mitochondrial oxygen consumptions in this observation. It has been reported that pathological stress activates the chromatin repressor complex containing HDAC to inhibit the transcription of Mhrt, a long noncoding RNA in the heart [57]. It would be interesting to define whether long non-coding transcripts would also involve the protective effects of HDAC inhibitors in the prevention of diabetic pathology. In this study, we utilized a high dose of STZ to induce the diabetic model. Because the dose of STZ that was used could be cytotoxic in the animal strain, this could be associated with the high mortality rate and abundant myocyte death during the development of diabetes. This could complicate the type 1 diabetic phenotype. This is a limitation for this observation.

\section{Conclusion}

Our study is the first to document that HDAC inhibition attenuated diabetic cardiomyopathy by improving ventricular function, attenuating remodeling, and suppressing cardiac hypertrophy. The protective effects of HDAC inhibition in the diabetic myocardium are closely associated with decreased apoptosis, stimulation of endogenous angiogenesis, an increase of anti-oxidant SOD1, and activations of GLUT1 acetylation and p38 phosphorylation. HDAC inhibition provides a novel approach to the treatment of patients with diabetic cardiomyopathy. Our study indicated that inhibition of HDACs effectively prevented cardiac dysfunction and cardiac remodeling in the diabetic heart, which is related to the promotion of angiogenesis, anti-apoptosis, and acetylation of GLUT1.

\section{Perspectives and translational implication}

Diabetes mellitus threatens to become a global health crisis; treatment of diabetes and its implications constitutes a major health care expenditure. A significant proportion of diabetic patients are known to develop diabetic cardiomyopathy, which is one of the leading causes of increased morbidity and mortality in patients with diabetes mellitus. HDAC regulates transcriptional changes and plays a critical role in mediating myocardial development and disease. From both a mechanistic and a translational point of view, the pathway identified in the present study will provide a strong foundation on which HDAC inhibition may serve as the novel therapeutic target to prevent the progression of diabetes-induced cardiac dysfunction. HDAC inhibitors are well tolerated in humans, and clinical trials investigating their efficacy in anti-cancer therapy are currently underway. Therefore, the therapeutic merit of HDAC inhibition in treating diabetic cardiomyopathy is potentially important. Exploration of the functional role of HDAC inhibition and evaluation of its clinical outcome will provide direct evidence to support a potential clinical implication.

\section{Additional file}

Additional file 1: Table S1. Echocardiographic parameters among the different groups. Table S2. Heart weight, body weight and heart/body ratio. Figure S1. Other HDAC isoforms in myocardium from STZ-treated mice and treatments. Figure S2. HDAC inhibition decreased the production of superoxide in myocardium from STZ-induced diabetic mice and different treatments.

\section{Abbreviations}

HDACs: histone deacetylases; HATs: histone acetyltransferases; DM: diabetic myocardium; STZ: streptozotocin; NaBu: sodium butyrate; LV: left ventricular; GLUT: glucose transporter type; LVID;d: left ventricular internal dimensiondiastole; LVID;s: left ventricular internal dimension-systole; EF: ejection fraction; FS: fraction shortening. 


\section{Authors' contributions}

YC, JD, YTZ, LZ Perform experiment; Data analysis. YC; JD and TZ Write manuscript; GL; SZ, GQ and TZ: Experimental designs. All authors read and approved the final manuscript.

\section{Author details}

${ }^{1}$ Department of Surgery, Boston University Medical School, Roger Williams Medical Center, Boston University, 50 Maude Street, Providence, RI 02908 , USA. ${ }^{2}$ Department of Medicine, Rhode Island Hospital, Brown University, Providence, RI, USA. ${ }^{3}$ Department of Ultrasound, Second Affiliated Hospital of Fujian Medical University, Quanzhou, Fujian, China. ${ }^{4}$ Feinberg Cardiovascular Research Institute, Northwestern University Feinberg School of Medicine, Chicago, USA.

\section{Acknowledgements}

The work is supported by the National Heart, Lung, and Blood Institute Grant (R01 HL089405 and R01 HL115265) and American Heart Association-National center $(0735458 \mathrm{~N})$

\section{Compliance with ethical guidelines}

\section{Competing interests}

The authors declare that they have no competing interests.

Received: 6 July 2015 Accepted: 18 July 2015

Published online: 07 August 2015

\section{References}

1. Garcia MJ, McNamara PM, Gordon T, Kannel WB (1974) Morbidity and mortality in diabetics in the Framingham population. Sixteen year followup study. Diabetes 23:105-111

2. (1999) Diabetes mellitus. A major risk factor for cardiovascular disease: a joint editorial statement by the American Diabetes Association; The National Heart, Lung, and Blood Institute; The Juvenile Diabetes Foundation International; The National Institute of Diabetes and Digestive and Kidney Diseases; and The American Heart Association., Circulation 100:1132-3

3. Zhao TC (2013) Glucagon-like peptide-1 (GLP-1) and protective effects in cardiovascular disease: a new therapeutic approach for myocardial protection. Cardiovasc Diabetol 12:90

4. Rubler S, Dlugash J, Yuceoglu YZ, Kumral T, Branwood AW, Grishman A (1972) New type of cardiomyopathy associated with diabetic glomerulosclerosis. Am J Cardiol 30:595-602

5. Carugo S, Giannattasio C, Calchera I, Paleari F, Gorgoglione MG, Grappiolo A et al (2001) Progression of functional and structural cardiac alterations in young normotensive uncomplicated patients with type 1 diabetes mellitus. J Hypertens 19:1675-1680

6. Itoh S, Ding B, Shishido T, Lerner-Marmarosh N, Wang N, Maekawa $\mathrm{N}$ et al (2006) Role of p90 ribosomal S6 kinase-mediated proreninconverting enzyme in ischemic and diabetic myocardium. Circulation 113:1787-1798

7. Gross ER, Hsu AK, Gross GJ (2007) Diabetes abolishes morphine-induced cardioprotection via multiple pathways upstream of glycogen synthase kinase-3beta. Diabetes 56:127-136

8. Roe ND, Thomas DP, Ren J (2011) Inhibition of NADPH oxidase alleviates experimental diabetes-induced myocardial contractile dysfunction. Diabetes Obes Metab 13:465-473

9. Kajstura J, Fiordaliso F, Andreoli AM, Li B, Chimenti S, Medow MS et al (2001) IGF-1 overexpression inhibits the development of diabetic cardiomyopathy and angiotensin II-mediated oxidative stress. Diabetes 50:1414-1424

10. Van Linthout S, Seeland U, Riad A, Eckhardt O, Hohl M, Dhayat N et al (2008) Reduced MMP-2 activity contributes to cardiac fibrosis in experimental diabetic cardiomyopathy. Basic Res Cardiol 103:319-327

11. Shiomi T, Matsusaka H, Hayashidani S, Suematsu N, Wen J, Kubota T et al (2003) Streptozotocin-induced hyperglycemia exacerbates left ventricular remodeling and failure after experimental myocardial infarction. J Am Coll Cardiol 42:165-172
12. Dong B, Yu QT, Dai HY, Gao YY, Zhou ZL, Zhang L et al (2012) Angiotensinconverting enzyme-2 overexpression improves left ventricular remodeling and function in a rat model of diabetic cardiomyopathy. J Am Coll Cardiol 59:739-747

13. Cheung $P$, Allis CD, Sassone-Corsi $P$ (2000) Signaling to chromatin through histone modifications. Cell 103:263-271

14. Strahl BD, Allis CD (2000) The language of covalent histone modifications. Nature 403:41-45

15. Luger K, Mader AW, Richmond RK, Sargent DF, Richmond TJ (1997) Crystal structure of the nucleosome core particle at $2.8 \mathrm{~A}$ resolution. Nature 389:251-260

16. Wang D, Fang C, Zong NC, Liem DA, Cadeiras M, Scruggs SB et al (2013) Regulation of acetylation restores proteolytic function of diseased myocardium in mouse and human. Mol Cell Proteomics 12:3793-3802

17. Kong Y, Tannous P, Lu G, Berenji K, Rothermel BA, Olson EN et al (2006) Suppression of class I and II histone deacetylases blunts pressure-overload cardiac hypertrophy. Circulation 113:2579-2588

18. Haberland M, Montgomery RL, Olson EN (2009) The many roles of histone deacetylases in development and physiology: implications for disease and therapy. Nat Rev Genet 10:32-42

19. Kee HJ, Sohn IS, Nam KI, Park JE, Qian YR, Yin Z et al (2006) Inhibition of histone deacetylation blocks cardiac hypertrophy induced by angiotensin II infusion and aortic banding. Circulation 113:51-59

20. Granger A, Abdullah I, Huebner F, Stout A, Wang T, Huebner T et al (2008) Histone deacetylase inhibition reduces myocardial ischemia-reperfusion injury in mice. FASEB J 22:3549-3560

21. Kao YH, Liou JP, Chung CC, Lien GS, Kuo CC, Chen SA et al (2013) Histone deacetylase inhibition improved cardiac functions with direct antifibrotic activity in heart failure. Int J Cardiol 168:4178-4183

22. Eom GH, Nam YS, Oh JG, Choe N, Min HK, Yoo EK et al (2014) Regulation of acetylation of histone deacetylase 2 by p300/CBP-associated factor/ histone deacetylase 5 in the development of cardiac hypertrophy. Circ Res 114:1133-1143

23. Zhang L, Qin X, Zhao Y, Fast L, Zhuang S, Liu P et al (2012) Inhibition of histone deacetylases preserves myocardial performance and prevents cardiac remodeling through stimulation of endogenous angiomyogenesis. J Pharmacol Exp Ther 341:285-293

24. Zhang LX, Zhao Y, Cheng G, Guo TL, Chin YE, Liu PY et al (2010) Targeted deletion of NF-kappaB p50 diminishes the cardioprotection of histone deacetylase inhibition. Am J Physiol Heart Circ Physiol 298:H2154-H2163

25. Zhao TC, Cheng G, Zhang LX, Tseng YT, Padbury JF (2007) Inhibition of histone deacetylases triggers pharmacologic preconditioning effects against myocardial ischemic injury. Cardiovasc Res 76:473-481

26. Chen HP, Denicola M, Qin X, Zhao Y, Zhang L, Long XL et al (2011) HDAC inhibition promotes cardiogenesis and the survival of embryonic stem cells through proteasome-dependent pathway. J Cell Biochem 112:3246-3255

27. Zhang L, Chen B, Zhao Y, Dubielecka PM, Wei L, Qin GJ et al (2012) Inhibition of histone deacetylase-induced myocardial repair is mediated by c-kit in infarcted hearts. J Biol Chem 287:39338-39348

28. Cox EJ, Marsh SA (2013) Exercise and diabetes have opposite effects on the assembly and O-GICNAc modification of the mSin3A/HDAC 1/2 complex in the heart. Cardiovasc Diabetol 12:101

29. Yu XY, Geng YJ, Liang JL, Lin QX, Lin SG, Zhang S et al (2010) High levels of glucose induce apoptosis in cardiomyocyte via epigenetic regulation of the insulin-like growth factor receptor. Exp Cell Res 316:2903-2909

30. Tsutsui H, Matsushima S, Kinugawa S, Ide T, Inoue N, Ohta Y et al (2007) Angiotensin II type 1 receptor blocker attenuates myocardial remodeling and preserves diastolic function in diabetic heart. Hypertens Res 30:439-449

31. Christensen DP, Dahllof M, Lundh M, Rasmussen DN, Nielsen MD, Billestrup N et al (2011) Histone deacetylase (HDAC) inhibition as a novel treatment for diabetes mellitus. Mol Med 17:378-390

32. Patel BM, Raghunathan S, Porwal U (2014) Cardioprotective effects of magnesium valproate in type 2 diabetes mellitus. Eur J Pharmacol 728:128-134

33. Cooper ME, El-Osta A (2010) Epigenetics: mechanisms and implications for diabetic complications. Circ Res 107:1403-1413

34. Asrih M, Steffens S (2013) Emerging role of epigenetics and miRNA in diabetic cardiomyopathy. Cardiovasc Pathol 22:117-125 
35. Regan TJ, Lyons MM, Ahmed SS, Levinson GE, Oldewurtel HA, Ahmad MR, Haider B (1977) Evidence for cardiomyopathy in familial diabetes mellitus. J Clin Invest 60:884-899

36. Busche MN, Walsh MC, McMullen ME, Guikema BJ, Stahl GL (2008) Mannose-binding lectin plays a critical role in myocardial ischaemia and reperfusion injury in a mouse model ofdiabetes. Diabetologia 51:1544-1551

37. Young ME, Wilson CR, Razeghi P, Guthrie PH, Taegtmeyer H (2002) Alterations of the circadian clock in the heart by streptozotocin-induced diabetes. J Mol Cell Cardiol 34:223-231

38. Hoit BD, Castro C, Bultron G, Knight S, Matlib MA (1999) Noninvasive evaluation of cardiac dysfunction by echocardiography in streptozotocininduced diabetic rats. J Card Fail 5:324-333

39. Gao Z, Yin J, Zhang J, Ward RE, Martin RJ, Lefevre M et al (2009) Butyrate improves insulin sensitivity and increases energy expenditure in mice. Diabetes 58:1509-1517

40. Zhang LX, DeNicola M, Qin X, Du J, Ma J, Zhao TY et al (2014) Specific inhibition of HDAC4 in cardiac progenitor cells enhances myocardial repairs. Am J Physiol Cell Physiol 307:C358-C372

41. Zhang CL, McKinsey TA, Chang S, Antos CL, Hill JA, Olson EN (2002) Class II histone deacetylases act as signal-responsive repressors of cardiac hypertrophy. Cell 110:479-488

42. Lieb W, Xanthakis V, Sullivan LM, Aragam J, Pencina MJ, Larson MG et al (2009) Longitudinal tracking of left ventricular mass over the adult life course: clinical correlates of short- and long-term change in the Framingham offspring study. Circulation 119:3085-3092

43. Taegtmeyer H, McNulty P, Young ME (2002) Adaptation and maladaptation of the heart in diabetes: part I: general concepts. Circulation 105:1727-1733

44. Li Y, Ma J, Zhu H, Singh M, Hill D, Greer PA et al (2011) Targeted inhibition of calpain reduces myocardial hypertrophy and fibrosis in mouse models of type 1 diabetes. Diabetes 60:2985-2994

45. Bojunga J, Nowak D, Mitrou PS, Hoelzer D, Zeuzem S, Chow KU (2004) Antioxidative treatment prevents activation of death-receptor- and mitochondrion-dependent apoptosis in the hearts of diabetic rats. Diabetologia 47:2072-2080

46. Shen E, Li Y, Li Y, Shan L, Zhu H, Feng Q et al (2009) Rac1 is required for cardiomyocyte apoptosis during hyperglycemia. Diabetes 58:2386-2395

47. Nakamura H, Matoba S, Iwai-Kanai E, Kimata M, Hoshino A, Nakaoka M et al (2012) p53 promotes cardiac dysfunction in diabetic mellitus caused by excessive mitochondrial respiration-mediated reactive oxygen species generation and lipid accumulation. Circ Heart Fail 5:106-115
48. Kuo WW, Wang WJ, Tsai CY, Way CL, Hsu HH, Chen LM (2013) Diallyl trisufide (DATS) suppresses high glucose-induced cardiomyocyte apoptosis by inhibiting JNK/NFKB signaling via attenuating ROS generation. Int J Cardiol 168:270-280

49. Zhang S, Liu H, Amarsingh GV, Cheung CC, Hogl S, Narayanan U et al (2014) Diabetic cardiomyopathy is associated with defective myocellular copper regulation and both defects are rectified by divalent copper chelation. Cardiovasc Diabetol 13:100

50. Hudlicka O, Brown M, Egginton S (1992) Angiogenesis in skeletal and cardiac muscle. Physiol Rev 72:369-417

51. Samuel SM, Thirunavukkarasu M, Penumathsa SV, Koneru S, Zhan L, Maulik G et al (2010) Thioredoxin-1 gene therapy enhances angiogenic signaling and reduces ventricular remodeling in infarcted myocardium of diabetic rats. Circulation 121:1244-1255

52. Khazaei M, Fallahzadeh AR, Sharifi MR, Afsharmoghaddam N, Javanmard SH, Salehi E (2011) Effects of diabetes on myocardial capillary density and serum angiogenesis biomarkers in male rats. Clinics (Sao Paulo) 66:1419-1424

53. Kainulainen $H$, Breiner $M$, Schurmann A, Marttinen A, Virjo A, Joost HG (1994) In vivo glucose uptake and glucose transporter proteins GLUT1 and GLUT4 in heart and various types of skeletal muscle from streptozotocin-diabetic rats. Biochim Biophys Acta 1225:275-282

54. Zhao T, Parikh P, Bhashyam S, Bolukoglu H, Poornima I, Shen YT et al (2006) Direct effects of glucagon-like peptide-1 on myocardial contractility and glucose uptake in normal and postischemic isolated rat hearts. J Pharmacol Exp Ther 317:1106-1113

55. Marciniak C, Marechal X, Montaigne D, Neviere R, Lancel S (2014) Cardiac contractile function and mitochondrial respiration in diabetes-related mouse models. Cardiovasc Diabetol 13:118

56. DeNicola M, Du J, Wang Z, Yano N, Zhang L, Wang Y et al (2014) Stimulation of glucagon-like peptide-1 receptor through exendin-4 preserves myocardial performance and prevents cardiac remodeling in infarcted myocardium. Am J Physiol Endocrinol Metab 307:E630-E643

57. Han P, Li W, Lin CH, Yang J, Shang C, Nurnberg ST et al (2014) A long noncoding RNA protects the heart from pathological hypertrophy. Nature 514:102-106

\section{Submit your next manuscript to BioMed Central and take full advantage of:}

- Convenient online submission

- Thorough peer review

- No space constraints or color figure charges

- Immediate publication on acceptance

- Inclusion in PubMed, CAS, Scopus and Google Scholar

- Research which is freely available for redistribution

Submit your manuscript at 